\title{
Head roll dependent variability of subjective visual vertical and ocular counterroll
}

\author{
Tarnutzer, A A ; Bockisch, C J ; Straumann, D
}

\begin{abstract}
We compared the variability of the subjective visual vertical (SVV) and static ocular counterroll (OCR), and hypothesized a correlation between the measurements because of their shared macular input. SVV and OCR were measured simultaneously in various whole-body roll positions [upright, 45 degrees right-ear down (RED), and 75 degrees RED] in six subjects. Gains of OCR were -0.18 (45 degrees RED) and -0.12 (75 degrees RED), whereas gains of compensation for body roll in the SVV task were -1.11 (45 degrees RED) and -0.96 (75 degrees RED). Normalized SVV and OCR variabilities were not significantly different $(\mathrm{P}>0.05)$, i.e., both increased with increasing roll. Moreover, a significant correlation $(\mathrm{R}(2)=0.80$, slope $=0.29)$ between SVV and OCR variabilities was found. Whereas the gain of OCR is different from the gain of SVV, trial-to-trial variability of OCR follows the same roll-dependent modulation observed in SVV variability. We propose that the similarities in variability reflect a common otolith input, which, however, is subject to distinct central processing for determining the gain of SVV and OCR.
\end{abstract}

DOI: https://doi.org/10.1007/s00221-009-1823-4

Posted at the Zurich Open Repository and Archive, University of Zurich

ZORA URL: https://doi.org/10.5167/uzh-18716

Journal Article

Published Version

Originally published at:

Tarnutzer, A A; Bockisch, C J; Straumann, D (2009). Head roll dependent variability of subjective visual vertical and ocular counterroll. Experimental Brain Research, 195(4):621-626.

DOI: https://doi.org/10.1007/s00221-009-1823-4 


\title{
Head roll dependent variability of subjective visual vertical and ocular counterroll
}

\author{
Alexander A. Tarnutzer • Christopher J. Bockisch • \\ Dominik Straumann
}

Received: 2 December 2008 / Accepted: 20 April 2009 / Published online: 5 May 2009

(C) Springer-Verlag 2009

\begin{abstract}
We compared the variability of the subjective visual vertical (SVV) and static ocular counterroll (OCR), and hypothesized a correlation between the measurements because of their shared macular input. SVV and OCR were measured simultaneously in various whole-body roll positions [upright, $45^{\circ}$ right-ear down (RED), and $75^{\circ}$ RED] in six subjects. Gains of OCR were $-0.18\left(45^{\circ}\right.$ RED) and $-0.12\left(75^{\circ} \mathrm{RED}\right)$, whereas gains of compensation for body roll in the SVV task were $-1.11\left(45^{\circ} \mathrm{RED}\right)$ and $-0.96\left(75^{\circ}\right.$ RED). Normalized SVV and OCR variabilities were not significantly different $(P>0.05)$, i.e., both increased with increasing roll. Moreover, a significant correlation $\left(R^{2}=0.80\right.$, slope $\left.=0.29\right)$ between SVV and OCR variabilities was found. Whereas the gain of OCR is different from the gain of SVV, trial-to-trial variability of OCR follows the same roll-dependent modulation observed in SVV variability. We propose that the similarities in variability reflect a common otolith input, which, however, is subject to distinct central processing for determining the gain of SVV and OCR.
\end{abstract}

Keywords Vestibular - Otolith organs - Eye movements . Perception

\footnotetext{
A. A. Tarnutzer $(\bowtie) \cdot$ C. J. Bockisch · D. Straumann Department of Neurology, Zurich University Hospital, Frauenklinikstrasse 26, 8091 Zurich, Switzerland

e-mail: alexander.tarnutzer@access.unizh.ch

C. J. Bockisch

Departments of Otorhinolaryngology and Ophthalmology,

Zurich University Hospital, Zurich, Switzerland
}

\section{Introduction}

Self orientation, relative to gravity, is determined by combining inputs from the vestibular system (utricular and saccular macula, semicircular canals or SCC), proprioception, and vision. Aligning a luminous line with the perceived earth-vertical, i.e., the subjective visual vertical (SVV), while being in a roll-tilted position requires compensation for body roll by rotation of the line away from the bodylongitudinal axis by an angle $\beta$ that represents estimated body roll. This compensation, i.e., position gain, is nearly perfect for small angles, but line settings tend to err toward over-compensation (E-effect) at moderate roll angles (Mueller 1916) and toward under-compensation (A-effect) at larger roll angles (Aubert 1861). It was suggested that Aand E-effects are a consequence of how sensory inputs are integrated into a unified percept of vertical (Mittelstaedt 1983).

Changes of head-roll orientation, relative to gravity, modulate the shear forces acting on the otoliths (Schoene 1964) and evoke reflexive torsional eye movements in the opposite direction, termed ocular counterroll (OCR) (Miller and Graybiel 1962). Under static conditions, OCR is predominantly driven by inputs from the otoliths (Fernandez et al. 1972; Tomko et al. 1981) and compensates only for a small percentage (5-25\%) of head roll (Diamond et al. 1979; Collewijn et al. 1985; Bockisch and Haslwanter 2001; Palla et al. 2006). OCR likely depends upon the integration of both utricular and saccular signals. Providing identical inter-aural shear to the otoliths but varying craniocaudal shear by applying centrifugation or static head roll, centrifugation was found to yield larger ocular torsion (OT) (MacDougall et al. 1999), supporting a saccular contribution to OT in humans. De Graaf et al. (1996), based on a variety of paradigms stimulating the otoliths, estimated that 
the utricular contribution to OCR is about three times greater than the saccular contribution. Saccular input to the equilibrium and to OT was also reported in cats (Tomko et al. 1981), squirrel monkeys (Fernandez et al. 1972) and rabbits (Maruta et al. 2008).

Subjective visual vertical variability increases with increasing head roll (Mittelstaedt 1983; De Vrijer et al. 2008 ) and peaks around $120^{\circ}-150^{\circ}$ (Schoene and Udo de Haes 1968; Udo de Haes 1970; Lechner-Steinleitner 1978). This pattern was explained by a "decreasing effectiveness" of the otolith organs with increasing roll (Schoene and Udo de Haes 1968; Lechner-Steinleitner 1978), though the contribution of other sensory systems and SVV decision processes cannot be discounted. Considering that estimating static head roll required for SVV (Schoene and Udo de Haes 1968) and OCR (Fernandez et al. 1972; Tomko et al. 1981) mainly originates from the otoliths (Miller et al. 1968), we hypothesize that a shared otolith input may be reflected in a significant correlation between the variabilities of SVV and OCR. In other words, we expect that OCR variability also increases with increasing head roll. Different patterns of SVV and OCR variability would suggest an extra-otolithic contribution to the SVV (Udo de Haes 1970). For example, the contribution of somatosensory inputs could vary with roll position for the SVV and OCR, or decision processes related to the SVV could produce changes in variability. Previous studies provided evidence both for (Haustein 1992) and against (Udo de Haes 1970) a rollangle dependent modulation of OCR variability. Since, in these studies subjects completed multiple measurements without changing their roll position and, therefore, allowed for adaptive mechanisms of OCR over time (Pansell et al. 2005), we repeated experiments by pseudorandomly changing the whole-body roll angle before each measurement. This refined protocol yielded OCR and SVV variabilities devoid of adaptation, which in turn could be correlated. We anticipate that variabilities of SVV and OCR differ, since the gain of compensatory SVV roll (close to -1 ) and the gain of compensatory torsional eye movements $(-0.05$ to -0.25$)$ are not in the same range. However, after normalizing the distinct noise levels of SVV and OCR, our hypothesis predicts similar variabilities of both parameters.

\section{Materials and methods}

\section{Subjects}

We studied seven healthy human subjects ( 2 females; $27-$ 43 years old). One subject had to be excluded due to poor OCR and SVV responses due to sleepiness. Informed consent of all subjects was obtained after full explanation of the experimental procedure. The protocol was approved by a local ethics committee and was in accordance with the ethical standards laid down in the 1964 Declaration of Helsinki for research involving human subjects.

\section{Experimental setup}

All experiments were performed on a motor-driven turntable (Acutronic, Jona, Switzerland). Subjects were seated upright and secured with a safety belt. The head was restrained in the natural straight ahead position with a thermoplastic mask. A turntable-fixed coil frame surrounded the head and generated three orthogonal magnetic fields. Three-dimensional movements of the right eye were recorded with a dual scleral search coil (Skalar Instruments, Delft, The Netherlands) at a frequency of $1,000 \mathrm{~Hz}$. To minimize torsional eye movement artifacts by mechanical interaction of the nasally exiting wire, modified search coils with the wire exiting inferiorly were used (Bergamin et al. 2002). An arrow with a length of $9.5^{\circ}$ was projected on a spherical surface situated $1.5 \mathrm{~m}$ in front of the subject's eyes. A short, $0.8^{\circ}$ long line bisected the arrow, providing a straight-ahead fixation point. The arrow roll orientation had a resolution of $0.1^{\circ}$. A remote control box allowed the subjects to rotate the arrow and to confirm the completion of each adjustment.

\section{Experimental protocol}

Recording sessions were limited to $\sim 40$ min due to possible corneal edema and blurred vision. We restricted the paradigm to a few discrete roll angles and obtained enough trials to calculate reliable trial-to-trial variabilities. To collect data at roll positions that were over- or under-compensated in the SVV-task (Aubert 1861; Mueller 1916), $45^{\circ}$ right-ear down (RED) and $75^{\circ}$ RED were chosen. With the exception of the luminous arrow or dot, the visual surrounding was completely dark. Before each trial, subjects were placed upright and were asked to look straight-ahead at a laser dot. The 3D eye position during this visual fixation was defined as the reference zero position. In total, 120 trials (40 trials each in all roll positions) were collected in a single session. In each trial, subjects were brought from upright to a roll position that was upright, $45^{\circ} \mathrm{RED}$, or $75^{\circ}$ RED with a constant turntable acceleration of $\pm 10^{\circ} / \mathrm{s}^{2}$. This resulted in peak turntable velocities of $\sim \pm 20 \%$ s $\left(45^{\circ}\right.$ position shifts) and $\sim \pm 26^{\circ} / \mathrm{s}$ ( $75^{\circ}$ position shifts), respectively. The arrow projection started $\sim 5 \mathrm{~s}$ after the turntable came to a full stop and the arrow starting position deviated pseudo-randomly between $28^{\circ}$ and $82^{\circ}$ clockwise (CW) or counterclockwise $(\mathrm{CCW})$ from the gravity vector. Rotations with accelerations above the threshold of the SCC 
influence errors in SVV (Jaggi-Schwarz and Hess 2003; Pavlou et al. 2003). To quantify the contribution of the SCC, we checked for post-rotatory torsional ocular drift and nystagmus in our paradigms. Average torsional eye velocity at the time subjects confirmed arrow adjustments was found to be small $(0.10 \pm 0.06 \% \mathrm{~s})$. Moreover, average deviations in horizontal and vertical eye positions at this moment were within $\pm 4.5^{\circ}$ from straight ahead, making eye torsion resulting from eye eccentricity within Listing's plane (Haustein 1989) unlikely.

\section{Data analysis}

Three-dimensional eye positions were computed and digitally filtered (Gaussian filter with a width of 51 samples, zero-phase forward and reverse filtering). $\mathrm{CW}$ torsion, as seen by the subject, was positive. Although we used modified search coils, small torsional coil slips were not totally excluded. To further reduce possible slip-related errors, we analyzed OT relative to the previous reference zero position determined before each trial. Average $( \pm 1$ standard deviation or SD) arrow roll orientations and torsional eye positions in different roll positions in a head-fixed reference frame were calculated. For the SVV, we determined the arrow roll angle $\beta_{S V V}$ which compensates the angle of whole-body roll and its gain. A gain of -1 indicates perfect compensation; a gain <-1 under-compensation (A-effect), and a gain $>-1$ over-compensation (E-effect). The same nomenclature is used for OCR, for which we determined OCR angle $\beta_{\mathrm{OCR}}$ and its gain. Data points were considered as outliers if they were more distant from the average than three SD. In total, $<0.5 \%$ of all data points were outliers and were discarded before statistical analysis.

Since both OCR and SVV variabilities depended on whole-body roll, and so were measured with error, principal component analysis (PCA) was chosen in order to evaluate for the proposed correlation. This procedure is equivalent to Orthogonal Linear Regression or Total Least Squares, which minimizes the perpendicular distances from the data points to the fitted model (Van Huffel and Vandewalle 1991). Multiple least square linear regression differs from PCA in that it implies that one variable, i.e., the independent variable, is known without error. Conversely, PCA appropriately adjusts for errors along all axes. This correlation method requires normalization, which is achieved by dividing individual values by the standard error of the whole data population. As a measure of the goodness of fit we provide the $R^{2}$ value. To estimate the sampling distribution of the slope of the fit obtained by PCA, we used bootstrapping to construct 1,000 resamples and calculated the 95\% confidence interval (CI). The correlation between SVV and OCR variability was considered significant whenever the CI did not include zero. To compensate for the distinct noise levels of SVV and OCR variability observed, we compared the normalized variability values, obtained by dividing individual values through the standard error of the whole data population. We used analysis of variance (ANOVA) with Tukey's correction for multiple comparisons of SVV and OCR at different roll angles. In some sections, the statistical analysis was solely based on paired $t$ tests with Holm's correction for multiple comparisons (Holm 1979).

\section{Results}

\section{Absolute values of SVV and OCR}

Since no statistically significant (ANOVA, $P>0.05$ ) main effect of the direction of arrow rotation was found for both SVV and OCR, trials with starting $\mathrm{CW}$ and $\mathrm{CCW}$ arrow orientation offsets were pooled. In upright position, SVV did not deviate significantly from earth-vertical $(t$ test, $\left.0.4 \pm 1.9^{\circ}, P>0.05\right)$. At $45^{\circ} \mathrm{RED}$, average ( $\pm 1 \mathrm{SD}$ ) compensatory angle $\beta_{\mathrm{SVV}}$ was $-50.0^{\circ}\left( \pm 6.5^{\circ}\right)$ relative to the whole-body roll orientation, indicating slight roll overcompensation with a position gain of -1.11 . At $75^{\circ}$ RED, average $\beta_{\mathrm{SVv}}$ was $-72.3^{\circ}\left( \pm 9.4^{\circ}\right)$, showing a tendency toward roll under-compensation and yielding a gain of -0.96 . At both $45^{\circ}$ and $75^{\circ} \mathrm{RED}, \beta_{\mathrm{SVv}}$ values of the subjects tested were not significantly $(t$ test, $P>0.05)$ different from perfect compensation.

In contrast to $\beta_{\mathrm{SVV}}$, the compensatory eye torsion $\beta_{\mathrm{OCR}}$, i.e., static OCR reached only a fraction of whole-body roll. At $45^{\circ}$ RED $\beta_{\mathrm{OCR}}$ averaged $-8.2^{\circ}$, yielding a gain of -0.18 . OCR was slightly increased at $75^{\circ}$ RED $\left(-8.8^{\circ}\right)$, which corresponds to a gain of 0.12 . At this whole-body roll position, the inter-individual $\mathrm{SD}$ was larger $\left(75^{\circ} \mathrm{RED}\right.$ : $1.1^{\circ} ; 45^{\circ}$ RED: $0.5^{\circ}$ ).

\section{Trial-to-trial variability of SVV and OCR}

Figure 1a shows the average trial-to-trial variability for both SVV and OCR as a function of whole-body roll. Since there was no significant (ANOVA, $P>0.05$ ) main effect of the direction of arrow rotation for both SVV and OCR, trials with $\mathrm{CW}$ and $\mathrm{CCW}$ arrow rotations were pooled. Absolute values of SVV and OCR variabilities were in different ranges, yielding SVV-to-OCR ratios of 3.5 ( $\left.45^{\circ} \mathrm{RED}\right)$ and 4.0 ( $\left.75^{\circ} \mathrm{RED}\right)$. For direct comparison of SVV and OCR, we performed normalization by dividing individual variability values by the standard error of the whole data population. Normalized average $( \pm 1 \mathrm{SD}) \mathrm{SVV}$ variability was significantly smaller (ANOVA, $P<0.001$ ) in upright position $(5.4 \pm 1.7)$ than in roll-tilted positions $\left(45^{\circ}\right.$ RED: $14.7 \pm 3.6 ; 75^{\circ}$ RED: $\left.16.8 \pm 4.4\right)$. Compared to upright 


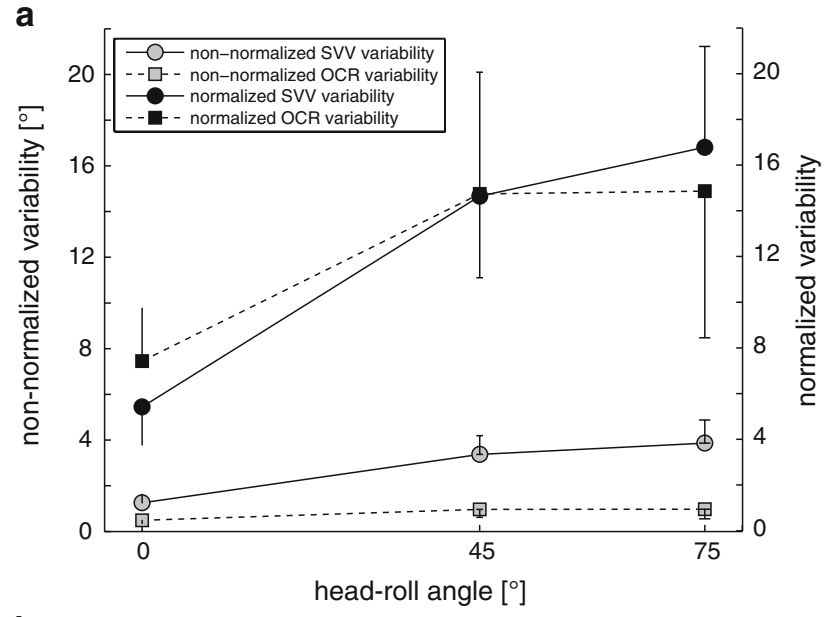

b

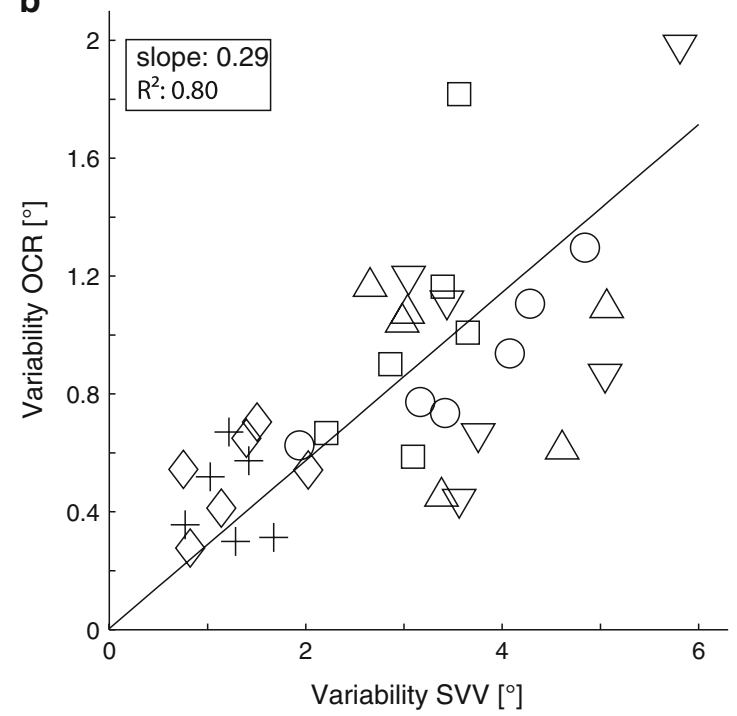

Fig. 1 a Average ( $\pm 1 \mathrm{SD})$ non-normalized (in gray) and normalized (in black) trial-to-trial variability of SVV (circles) and OCR (squares) plotted against head-roll angle. b Non-normalized trial-to-trial SVV variabilities are plotted against non-normalized variabilities of OCR. Symbols (CW/CCW arrow rotations): diamonds/plus upright position, circles/squares $45^{\circ} \mathrm{RED}$, triangles/inverted triangles $75^{\circ}$ RED. Black line linear regression

(7.5 \pm 2.3 ), normalized OCR variability was significantly (ANOVA, $P<0.001)$ larger both at $45^{\circ}$ RED $(14.8 \pm 5.3)$ and $75^{\circ}$ RED $(14.9 \pm 6.4)$. After normalization, variability values of SVV and OCR were found to be of similar magnitude. Moreover, the difference between SVV and OCR variabilities at a given whole-body roll angle was not significantly different from zero ( $t$ test, Holm's corrected, $P>0.05$ ).

To explore the hypothesis that the intra-individual trialto-trial variabilities of OCR and SVV may rely on a common otolith input, OCR variabilities were plotted against SVV variabilities in Fig. 1b. The goodness-of-fit using PCA was high $\left(R^{2}=0.80\right)$; the slope of the linear regression was 0.29 (95\% CI $0.22-0.38)$, indicating that the variability in SVV is scaled by a factor of $\sim 3$ relative to the OCR variability.

\section{Discussion}

Increasing whole-body roll resulted in increasing trialto-trial variabilities of both the SVV and OCR. The high correlation between these variabilities and the fact that, after normalization, they were not significantly different at given roll positions support our hypothesis of a common otolith signal driving both SVV and OCR and their trialto-trial variability. Our observations are consistent with an experimental human study by Haustein (1992), in which a significant decrease in OCR precision at $90^{\circ}$ ear-down position relative to upright was reported. Conversely, our findings are in contrast to findings by Udo de Haes (1970), who reported no modulation of OCR variability, but increasing SVV variability with increasing head roll. Differences in the experimental setup may explain this discrepancy of OCR results. Whereas Udo de Haes placed two subjects in prone position with their head bent back, we roll-tilted subjects en-bloc while sitting. However, no direct comparison of elicited OCR under these two conditions is available. As shown in Figure 6 of the publication, both the average variability and the spread of individual trial-to-trial OCR variabilities in roll-tilted positions were increased compared to upright position. Possibly, the considerable spread and the small number of subjects included $(n=2)$ by Udo de Haes masked the systematic roll-dependent modulation of OCRvariability that was found in our study. Furthermore, Udo de Haes ran repetitive trials without changing the subject's roll position between trials at a given roll position, whereas we brought subjects back to upright position after each trial avoiding drift of OCR over time, which has been described by others (Pansell et al. 2005).

Subjective visual vertical and OCR, although relying on shared otolith input, depend on different central circuits. Perception of vertical emerges from integrating multiple sensory inputs, including proprioceptive, visual, and vestibular input within vestibular cortical areas (Brandt and Dieterich 1999; Angelaki and Cullen 2008). OCR is generated by brainstem circuits (Crawford et al. 2003) mainly relying on vestibular input. In case of bilateral vestibular loss, OCR is reduced (Miller et al. 1968) and the contribution of extra-vestibular sensors to the residual OCR is greater (Miller and Graybiel 1963; Krejcova et al. 1971; De Graaf et al. 1992). In paradigms investigating the contribution of otolith signals to verticality perception, proprioceptive input is usually also available. However, verticality perception is not systematically altered by water immersion, which greatly reduces proprioceptive input (Graybiel et al. 1968; Jarchow and Mast 1999). On the other hand, 
bilateral vestibular deficits lead to a shift from E- to A-effects at small roll angles (Graybiel et al. 1968) and to an increase of the A-effect at larger roll angles (Miller et al. 1968; Bronstein et al. 1996), while impaired somatosensory function decreases the A-effect (Yardley 1990; Anastasopoulos et al. 1999; Bronstein 1999). Based on these observations, vestibular cues may play a central role in counterbalancing visually and proprioceptive mediated biases on the perception of verticality (Bronstein et al. 1996).

The roll-angle dependent modulation of both gravity perception and OCR has been investigated previously, but the reported relationships between perceived visual horizontal/vertical and OCR are contradictory. On the one hand, both Mast (2000) and Merker and Held (1981) failed to show a relationship between the magnitudes of OCR and SVV. Furthermore, Miller et al. (1968) demonstrated E-and A-effects in patients with bilateral vestibular loss (i.e., whose eyes do not tort), again suggesting that SVV and OCR modulate independently. On the other hand, De Graaf et al. (1992), Wade and Curthoys (1997) and Goonetilleke et al. (2008) reported a correlation between OCR and the subjective visual horizontal in healthy human subjects. In comparison to the modulation of the compensatory angle in SVV, however, both the gain and the rollangle dependent modulations of OCR are clearly distinct as observed here and reported previously (Udo de Haes 1970; Mast 2000).

By studying SVV and OCR over a larger range of roll angles, differences in central processing between SVV and OCR become evident as roll over-compensation in SVV switches to roll under-compensation around $60^{\circ}$ wholebody roll (Aubert 1861; Howard 1982; Van Beuzekom and Van Gisbergen 2000), whereas OCR increases further and peaks around $90^{\circ}$ whole-body roll, showing an approximately sinusoidal modulation (Udo de Haes 1970; Palla et al. 2006). Despite this difference in central processing, the common otolith input remains reflected in similar rolldependent SVV and OCR variabilities.

Acknowledgments The authors thank Albert Züger for technical assistance, Itsaso Olasagasti for statistical advice and Thomas Haslwanter for critically reading the manuscript. Funding was provided by the Swiss National Science Foundation (3200B0-105434), the Betty and David Koetser Foundation for Brain Research, Zurich, Switzerland, and the Center of Integrative Human Physiology, University of Zurich, Switzerland.

\section{References}

Anastasopoulos D, Bronstein A, Haslwanter T, Fetter M, Dichgans J (1999) The role of somatosensory input for the perception of verticality. Ann N Y Acad Sci 871:379-383

Angelaki DE, Cullen KE (2008) Vestibular system: the many facets of a multimodal sense. Annu Rev Neurosci 31:125-150
Aubert H (1861) Eine scheinbare bedeutende Drehung von Objekten bei Neigung des Kopfes nach rechts oder links. Virchows Arch 20:381-393

Bergamin O, Bizzarri S, Straumann D (2002) Ocular torsion during voluntary blinks in humans. Invest Ophthalmol Vis Sci 43:34383443

Bockisch CJ, Haslwanter T (2001) Three-dimensional eye position during static roll and pitch in humans. Vis Res 41:2127-2137

Brandt T, Dieterich M (1999) The vestibular cortex. Its locations, functions, and disorders. Ann N Y Acad Sci 871:293-312

Bronstein AM (1999) The interaction of otolith and proprioceptive information in the perception of verticality. The effects of labyrinthine and CNS disease. Ann N Y Acad Sci 871:324-333

Bronstein AM, Yardley L, Moore AP, Cleeves L (1996) Visually and posturally mediated tilt illusion in Parkinson's disease and in labyrinthine defective subjects. Neurology 47:651-656

Collewijn H, van der Steen J, Ferman L, Jansen TC (1985) Human ocular counterroll: assessment of static and dynamic properties from electromagnetic scleral coil recordings. Exp Brain Res 59:185-196

Crawford JD, Tweed DB, Vilis T (2003) Static ocular counterroll is implemented through the 3-D neural integrator. J Neurophysiol 90:2777-2784

De Graaf B, Bekkering H, Erasmus C, Bles W (1992) Influence of visual, vestibular, cervical, and somatosensory tilt information on ocular rotation and perception of the horizontal. J Vestib Res 2:15-30

De Graaf B, Bos JE, Groen E (1996) Saccular impact on ocular torsion. Brain Res Bull 40:321-326

De Vrijer M, Medendorp WP, Van Gisbergen JA (2008) Shared computational mechanism for tilt compensation accounts for biased verticality percepts in motion and pattern vision. J Neurophysiol 99:915-930

Diamond SG, Markham CH, Simpson NE, Curthoys IS (1979) Binocular counterrolling in humans during dynamic rotation. Acta Otolaryngol 87:490-498

Fernandez C, Goldberg JM, Abend WK (1972) Response to static tilts of peripheral neurons innervating otolith organs of the squirrel monkey. J Neurophysiol 35:978-987

Goonetilleke SC, Mezey LE, Burgess AM, Curthoys IS (2008) On the relation between ocular torsion and visual perception of line orientation. Vis Res 48:1488-1496

Graybiel A, Miller EF 2nd, Newsom BD, Kennedy RS (1968) The effect of water immersion on perception of the oculogravic illusion in normal and labyrinthine-defective subjects. Acta Otolaryngol 65:599-610

Haustein W (1989) Considerations on Listing's law and the primary position by means of a matrix description of eye position control. Biol Cybern 60:411-420

Haustein W (1992) Head-centric visual localization with lateral body tilt. Vis Res 32:669-673

Holm S (1979) A simple sequentially rejective multiple test procedure. Scand J Stat 6:65-70

Howard IP (1982) Human visual orientation. Wiley, New York

Jaggi-Schwarz K, Hess BJ (2003) Influence of dynamic tilts on the perception of earth-vertical. Exp Brain Res 149:340-350

Jarchow T, Mast FW (1999) The effect of water immersion on postural and visual orientation. Aviat Space Environ Med 70:879-886

Krejcova H, Highstein S, Cohen B (1971) Labyrinthine and extra-labyrinthine effects on ocular counter-rolling. Acta Otolaryngol $72: 165-171$

Lechner-Steinleitner S (1978) Interaction of labyrinthine and somatoreceptor inputs as determinants of the subjective vertical. Psychol Res 40:65-76

MacDougall HG, Curthoys IS, Betts GA, Burgess AM, Halmagyi GM (1999) Human ocular counterrolling during roll-tilt and centrifugation. Ann N Y Acad Sci 871:173-180 
Maruta J, Raphan T, Simpson JI, Cohen B (2008) Vertical (Z-axis) acceleration alters the ocular response to linear acceleration in the rabbit. Exp Brain Res 185:87-99

Mast F (2000) Does the world rock when the eyes roll? Swiss J Psychol 59:89-101

Merker BH, Held R (1981) Eye torsion and the apparent horizon under head tilt and visual field rotation. Vis Res 21:543-547

Miller EF, Graybiel A (1962) Counterrolling of the human eyes produced by head tilt with respect to gravity. Acta Otolaryngol 54:479-501

Miller AD, Graybiel A (1963) A comparison of ocular counter-rolling movements between normal persons and deaf subjects with bilateral labyrinthine defects. Ann Otol 72:885-893

Miller EF 2nd, Fregly AR, Graybiel A (1968) Visual horizontal-perception in relation to otolith-function. Am J Psychol 81:488-496

Mittelstaedt H (1983) A new solution to the problem of the subjective vertical. Naturwissenschaften 70:272-281

Mueller GE (1916) Ueber das Aubertsche Phaenomenon. Z Psychol Physiol Sinnesorg 49:109-246

Palla A, Bockisch CJ, Bergamin O, Straumann D (2006) Dissociated hysteresis of static ocular counterroll in humans. J Neurophysiol 95:2222-2232

Pansell T, Tribukait A, Bolzani R, Schworm HD, Ygge J (2005) Drift in ocular torsion during sustained head tilt. Strabismus 13:115-121
Pavlou M, Wijnberg N, Faldon ME, Bronstein AM (2003) Effect of semicircular canal stimulation on the perception of the visual vertical. J Neurophysiol 90:622-630

Schoene H (1964) On the role of gravity in human spatial orientation. Aerosp Med 35:764-772

Schoene H, Udo de Haes H (1968) Perception of gravity-vertical as a function of head and trunk position. Z Vgl Physiol 60:440-444

Tomko DL, Peterka RJ, Schor RH (1981) Responses to head tilt in cat eighth nerve afferents. Exp Brain Res 41:216-221

Udo de Haes HA (1970) Stability of apparent vertical and ocular countertorsion as a function of lateral tilt. Percept Psychophys 8:137142

Van Beuzekom AD, Van Gisbergen JA (2000) Properties of the internal representation of gravity inferred from spatial-direction and body-tilt estimates. J Neurophysiol 84:11-27

Van Huffel S, Vandewalle J (1991) The total least squares problem. Computational aspects and analysis. Society for Industrial and Applied Mathematics, Philadelphia

Wade SW, Curthoys IS (1997) The effect of ocular torsional position on perception of the roll-tilt of visual stimuli. Vis Res 37:10711078

Yardley L (1990) Contribution of somatosensory information to perception of the visual vertical with body tilt and rotating visual field. Percept Psychophys 48:131-134 